\title{
RESPOND - A PATIENT-CENTRED PROGRAMME TO PREVENT SECONDARY FALLS IN OLDER PEOPLE PRESENTING TO THE EMERGENCY DEPARTMENT WITH A FALL: PROTOCOL FOR A MIXED METHODS PROGRAMME EVALUATION
}

\section{Authors}

Morris $R L^{1}$, Brand $C A^{1,2}$, Ayton $D^{1}$, Hill $K D^{3}$, Redfern $J^{4}$, Nyman $S R^{5}$, Lowthian $\mathrm{A}^{1}$, Hill $A \mathrm{M}^{6}$, Etherton-Beer $C D^{7,8,9}$, Flicker $L^{7,8,9}$, Hunter $\mathrm{P}^{10}$, Barker $\mathrm{AL}^{1}$

\section{Author affiliations}

${ }^{1}$ Health Service Research Unit, Department of Epidemiology and Preventive Medicine, Monash University, Melbourne, Victoria, Australia

${ }^{2}$ Melbourne EpiCentre, University of Melbourne and Melbourne Health, Melbourne, Victoria, Australia

${ }^{3}$ School of Physiotherapy and Exercise Science, Curtin University, Perth, Western Australia

${ }^{4}$ The George Institute for Global Health, Sydney Medical School, University of Sydney, Camperdown, NSW, Australia

${ }^{5}$ Bournemouth University Dementia Institute and Department of Psychology, Faculty of Science and Technology, Bournemouth University, Poole, Dorset, UK

${ }^{6}$ School of Physiotherapy, The University of Notre Dame Australia, Fremantle, Western Australia, Australia

${ }^{7}$ University of Western Australia, Perth, Western Australia, Australia

${ }^{8}$ Geriatric Medicine, Royal Perth Hospital, Perth, Western Australia, Australia

${ }^{9}$ Harry Perkins Institute for Medical Research, Perth, Western Australia, Australia

${ }^{10}$ Alfred Health, Melbourne, Victoria, Australia 


\section{ABSTRACT}

\section{Background}

Programme evaluations conducted alongside randomised controlled trials (RCTs) have potential to enhance understanding of trial outcomes. This paper describes a multi-level programme evaluation to be conducted alongside an RCT of a falls prevention programme (RESPOND).

\section{Objectives}

1) To conduct a process evaluation in order to identify the degree of implementation fidelity and associated barriers and facilitators.

2) To evaluate the primary intended impact of the programme: participation in fall prevention strategies, and the factors influencing participation.

3) To identify the factors influencing RESPOND RCT outcomes: falls, fall injuries and ED representations.

\section{Methods/ Design}

Five hundred and twenty eight community-dwelling adults aged $60-90$ years presenting to two EDs with a fall will be recruited and randomly assigned to the intervention or standard care group. All RESPOND participants and RESPOND clinicians will be included in the evaluation. A mixed methods design will be used and a programme logic model will frame the evaluation. Data will be sourced from interviews, focus groups, questionnaires, clinician case notes, recruitment records, participantcompleted calendars, hospital administrative datasets, and audio-recordings of intervention contacts. Quantitative data will be analysed via descriptive and inferential statistics and qualitative data will be interpreted using thematic analysis.

\section{Discussion}


The RESPOND programme evaluation will provide information about contextual and influencing factors related to the RCT outcomes. The results will assist researchers, clinicians, and policy makers to make decisions about future falls prevention interventions. Insights gained are likely to be transferable to preventive health programmes for a range of chronic conditions.

\section{BACKGROUND}

Falls are a serious problem among community-dwelling older people and represent the leading cause of emergency department (ED) presentations for older adults [1]. Following an ED presentation for a fall, up to half of cases will experience subsequent falls, often resulting in detrimental physical and psychological consequences [2-5]. Various falls prevention approaches have reduced falls within the clinical trial setting [6]. However, there was a significant increase in age-standardised falls related hospitalisation rates for older people from 1999-00 to 2010-11, according to Australian data [7]. In addition, a Finnish study showed that age-adjusted fall related fatality rates for people aged 65 and over more than doubled between 1971 and 2002 [8]. These upward trends suggest that favourable trial results are not being sufficiently translated to practice.

Falls are often the result of a complex mix of physiological, medical, behavioural and environmental risk factors [9]. Furthermore, individual characteristics, such as socio-demographic factors, are also associated with risk of falling [10]. Therefore, effective falls risk management is a multi-component process, with best practice guidelines recommending early screening to detect risk factors, and implementation of tailored interventions, taking into account individual preferences, in order to address the necessary changes [11-13].

Key components influencing the success or failure of a programme are the rate of participation in and adherence to recommended falls prevention strategies among those receiving the intervention [11]. Adherence to multifactorial interventions has varied, ranging from $28-95 \%$ for individual components [14]. Lack of perceived personal relevance may partially explain poor participation 
rates, and has been expressed by up to $34 \%$ of older adults when provided with details of evidencebased falls prevention strategies [15]. Conversely, acceptability of interventions, including perceived relevance and benefit, and involvement in decision-making, has been shown to facilitate participation [16]. In addition, health literacy contributes to the capacity of an individual to partake in preventative health programmes [17]. As $50 \%$ of older Australians are likely to have inadequate health literacy [18], this may be a substantial factor related to participation in falls prevention strategies. Given the number of inter-related factors involved, it is often difficult to identify the key individual characteristics, participatory factors, and programme components responsible for facilitating or inhibiting a reduction in falls from clinical trial results alone.

Understanding of trial results can be enhanced by conducting a programme evaluation $[19,20]$. Evaluations can be conducted on a number of levels including process (the degree of, and factors that influence, implementation fidelity) $[21,22]$; impact (changes in specific participant behaviours, knowledge or skills) [23]; and outcome (whether or not a programme achieved its goals, and why) [24]. Comprehensive programme evaluations are especially pertinent for multicentre trials where there is a risk that the same programme may be implemented and received in different ways [19]. However, despite the value of conducting comprehensive programme evaluations alongside falls prevention trials, there is limited evidence of this occurring.

Elements of process evaluation have been reported alongside three RCTs of falls prevention programmes targeting cognitively intact older adults who have presented to the ED with a fall $[3,25$, 26]. None of the three trials demonstrated a significant reduction in falls between the intervention and standard care groups. However, evaluation of process factors allowed for some explanation of the trial results. Two of the studies reported adherence to falls prevention activities, with comparison between the intervention and control groups $[3,26]$. A referral-based intervention reported that adherence was highest for occupational therapy, physiotherapy and podiatry, and lowest for written and oral advice. In addition, control group contamination included $17 \%$ of 
standard care participants referred to physiotherapy and $4 \%$ to falls clinics [3]. Similarly, a Dutch study involving a geriatric assessment and multifactorial intervention reported that control group contamination was a possible factor influencing trial results [26]. In contrast, a process evaluation of a Dutch version of the successful British PROFET trial did not report participation in falls prevention activities for the control group, despite discussing the possibility that the lack of contrast between groups may have been a factor explaining the lack of favourable trial results [25]. However, the evaluation did comprise a number of additional process factors, allowing the authors to conclude that although the multidisciplinary programme was largely implemented according to protocol, and was acceptable to those delivering and receiving the program. Lack of effectiveness was potentially due to the relatively low number of referrals and recommendations ensuing from the suggestion to contact their GP for on-going management.

No impact evaluations of RCTs of falls prevention programs targeting older adults presenting to the ED with a fall have been conducted. However, a non-randomised pre-test post-test study evaluated the impact of peer-presented education sessions on falls-related attitude, knowledge and behaviour of older people [23]. The study demonstrates the value of conducting an impact evaluation, as a number of recommendations were made for effective targeting of falls prevention programmes. Evaluations of factors associated with RCT outcomes are also not evident in the current literature related to falls prevention programs targeting older people presenting to the ED with a fall. Identification of associations between certain participant characteristics, and trial outcomes can provide insight into which sub-groups of participants the intervention is most, and least, effective for. One German RCT of an intervention comprising a geriatric assessment and home visit, conducted a sub-group analysis and found that the intervention was most effective for participants who reported having had two or more falls during the year before recruitment into the study [27]. Although falls history is an important factor to consider, evaluation of a number of other health status, sociodemographic and health literacy factors, in relation to trial outcomes, may also provide deeper understanding of trial outcomes $[10,17,18]$. 
This paper describes a mixed methods process, impact, and outcome evaluation to be conducted alongside an RCT of a falls prevention programme - RESPOND. RESPOND is a patient-centred intervention designed to improve older persons' participation in falls prevention activities through delivery of patient-centred education and behaviour change strategies. The proposed evaluation intends to provide insight into the contextual and influencing factors related to the RESPOND RCT outcomes. Results of this study will be applicable to other falls prevention programmes, as well as a range of chronic conditions where similar preventive management styles are indicated.

\section{OBJECTIVES}

\section{For the process evaluation we will:}

a) Assess the degree to which RESPOND was implemented as planned.

b) Identify barriers and facilitators to implementation from the perspectives of those delivering and receiving the intervention.

\section{For the impact evaluation we will:}

a) Identify whether RESPOND increases participation in falls prevention strategies, and factors influencing participation, among the intervention group, compared with standard care.

b) Determine the degree to which participant characteristics and RESPOND programme factors are associated with participation in falls prevention strategies, among the intervention group.

\section{For the outcome evaluation we will:}

a) Determine the degree to which participant characteristics, participatory and RESPOND programme factors influence falls, fall-injuries and ED re-presentations.

\section{METHODS/ DESIGN}




\section{Study design}

Overview and purpose of logic model

The evaluation will be conducted alongside an RCT of the RESPOND programme and will apply a convergent parallel mixed methods design [28]. Data collected as part of the RCT will be utilised in addition to data collected specifically for programme evaluative purposes. A logic model (Figure 1) that outlines each component of the RESPOND programme was mapped as a framework to guide and inform the evaluation. The model articulates relationships between inputs (resources available for the programme), activities conducted with these resources, outputs (products of the programme activities), impacts (specific changes in participants' behaviour), and outcomes (fundamental change occurring as a result of the programme).

Levels of evaluation and how they relate to the logic model

Three levels of evaluation will be conducted: (1) process, (2) impact, and (3) outcome. The process evaluation relates to implementation fidelity and corresponds with the inputs, activities and outputs in the logic model. The impact evaluation focuses on factors related to achieving the primary intended behavioural change: increased participation in falls prevention strategies. The outcome evaluation will identify sub-groups for which the RESPOND programme is most and least effective in terms of reducing falls, fall-injuries and ED re-presentations. The impact and outcome evaluations correspond with their respective columns in the logic model. The evaluation plan is summarised in Table 1.

\section{RESPOND RCT}

Study design

Details of the RESPOND RCT are described elsewhere [29]. In summary, a single-blind multicentre RCT of the RESPOND falls prevention programme, compared with standard care, will be conducted. 
Participants and setting

All participants in the RESPOND RCT will contribute to the program evaluation. The RCT will recruit 528 community-dwelling persons aged 60-90 years who present over a 12-month period to two tertiary referral EDs in Perth and Melbourne, Australia, with a fall and who are planned to be discharged directly home from the hospital within 72 hours.

RESPOND RCT outcomes and data collection

A number of outcomes will be reported for the RESPOND RCT. However, for the purpose of the program evaluation, factors related to only three trial outcomes will be analysed. These outcomes are falls, fall injuries and ED re-presentations per person-year in the 12 months after recruitment. A fall is defined as per the World Health Organisation: "an event resulting in a person coming to rest inadvertently on the ground, floor or other lower level"[30]. A fall injury is any physical harm resulting from a fall.

Hospital administrative data will be audited to determine the number of ED presentations that occur during the 12-month follow-up. Participants in both groups of the trial will complete monthly calendars documenting details of any falls, fall injuries, and ED presentations on a daily basis. All participants will receive a monthly telephone call from a RESPOND outcome assessor to verify information recorded on calendars. The outcome assessors will be blinded to group allocation.

The RESPOND intervention The key inputs, activities and intended outputs of the RESPOND programme are summarised in the logic model (Figure 1). The focus of RESPOND is the reinforcement of positive health messages and 
participant-centred care in order to optimise participant engagement and participation in the programme.

Planned dosage of intervention

The dosage according to protocol is an initial 45 minute face-to-face session within two weeks of ED discharge, with the first coaching phone call made within two weeks of the initial visit and the second within three months. Remaining phone calls will occur at intervals that allow progress towards goals. There will be a minimum of two follow-up phone calls with each call lasting approximately 45 minutes. Each participant will receive an average of 10 hours of coaching over a six month period.

\section{Comparator group}

The comparator group will continue to receive standard care from all health professionals involved in their management within the ED and primary care setting during the 12-month follow up.

\section{Objective 1: Process evaluation}

a) The degree to which RESPOND was implemented as planned

Assessment of implementation fidelity aims to document how the intervention is delivered and received, and compare this with intended implementation. For the purpose of this objective, the domains to be evaluated are: the reach, delivery of (in terms of content and dosage), and exposure to the RESPOND intervention.

Reach refers to the proportion of intended target audience that participate in an intervention [31]. Hospital admitted episode and ED administrative data will be audited to identify the number of potentially eligible study participants and reported as an essential part of the RESPOND RCT 
outcome paper. The process evaluation will add to this by identifying the proportion of eligible participants who declined to participate in the trial and the reasons stated for declining. This includes differentiation between declining to participate in a research project and declining to participate in a falls prevention program. The primary reason stated by patients for declining to participate is also captured. This information will be collected by RESPOND recruiters. Reasons expressed for exiting the study prematurely will also be collected by RESPOND clinicians and outcome assessors as appropriate. All RESPOND recruiter, clinician and outcome assessor assessments and notes will be recorded on a password-protected specifically designed RESPOND database.

Evaluation of delivery will be divided into two sub-categories: content and dosage. Content refers to the delivery of each individual component of the RESPOND programme tailored to the individual participant. This includes the provision of education related to risk factors and their management, application of motivational interviewing techniques, shared decision making leading to choice of modules and goal setting, and coordination of referrals to appropriate community services (as per 'activities' and 'outputs' in Figure 1). Clinician-participant contacts will be audio-recorded, where written consent has been obtained, in order to evaluate the proportion of key RESPOND elements delivered. Ten percent of randomly selected intervention audio-recordings will be used for analysis. The degree of participatory decision-making will be evaluated by applying the Rochester Participatory Decision-Making Scale (RPAD) [32]. This tool relates closely to the focus of RESPOND as a patient-centred program and includes items such as the clinician clearly explaining the relevant issues, discussing uncertainties, clarifying agreement, examining barriers, and asking open ended questions. This tool has been found to be valid and reliable in a study of physician-patient communication with primary care physicians [32] . Other RESPOND components, such as provision of education and application of motivational interviewing techniques will be evaluated using qualitative methods (see analysis for details). The proportion and type of discussions that occur beyond the 
scope of RESPOND during the intervention contacts will be also be evaluated through analysis of the audio-recordings.

Dosage of intervention delivered will be evaluated for all intervention participants and compared to the planned dosage of delivery (detailed above). This will include information related to the timing, frequency and total number of intervention contacts made by the RESPOND clinician per participant, as well as total duration of participation in the intervention (maximum 6 months). Dosage data will be obtained from clinician records.

Exposure refers to the extent to which the intervention participants actively engage with and act on agreed recommendations [31]. Data collection will include the number and type of modules chosen, the number and type of goals chosen, and management strategies chosen to address the goals. Participant-reported achievement of agreed actions to address goals will be captured by clinicians and recorded on the RESPOND database for all intervention participants.

b) Barriers and enablers to implementation

In order to understand the reasons for the degree of implementation fidelity established above, barriers and facilitators will be identified from the perspectives of those delivering and receiving the intervention. This will include exploring domains such as acceptability of the programme content, including the modules and written and verbal education provided; programme delivery in terms of dosage and delivery mode (combination of home visit and telephone contacts); and the patientcentred health-coaching delivery style. Perceived benefit and perceived relevance of the RESPOND programme will also be evaluated.

Data will be collected from a number of sources. As part of the intervention delivery, clinicians will ask participants to identify barriers and facilitators to achieving RESPOND goals. This will be recorded in clinician notes, in the form of 'tick box' options including commonly identified barriers and 
facilitators [16]. Additional free text options will ensure barriers and enablers beyond preanticipated responses are captured.

In addition, all intervention participants will receive a questionnaire on completion of the RESPOND programme (6 months from commencement). This will seek feedback related to evaluation domains including barriers and facilitators to participation in the RESPOND programme, acceptability of the content, dosage, delivery mode, and perceived benefit and relevance of the programme. The survey instrument will be developed by the research team and will include a series of statements with fivepoint Likert scale response options (strongly agree to strongly disagree, with neutral as a central point). Additional free text options will be included in the questionnaire. The questionnaire will be posted or emailed (depending on the preferred communication method of the participant identified at recruitment). The timing of the questionnaire aims to reduce the potential for recall bias.

RESPOND clinicians will be individually interviewed at 12 months using a semi-structured interview template in order to ascertain information related to their experience delivering the RESPOND programme. Evaluation domains that will be explored will be similar to those explored in the intervention participant questionnaires to allow for triangulation of evaluation constructs. In addition, opinions related to the content, timing and frequency of RESPOND training and support will be sought. All consenting RESPOND clinicians employed throughout the trial period (a minimum of five) will be included in the programme evaluation. As data from the perspective of those delivering the intervention is qualitative, this sample size is considered to be sufficient for the purpose of analyses.

RESPOND intervention participants who have completed the programme will be invited to take part in a focus group. Focus groups consisting of 8-10 participants will be conducted at both trial sites using a semi-structured template exploring the evaluation domains included in the participant questionnaires. Quota sampling will ensure a broad range of participants according to sociodemographic and health status factors and will consider (but not be limited to): Gender, age and falls 
risk profile. Falls risk profile will be determined and categorised as mild, moderate, or high as per the Falls Risk for Older People - Community setting (FROP-Com) which will be administered to all participants at baseline $[33,34]$. The sample size will be determined once the focus groups have been completed and saturation of themes has been reached. In the instance of certain sociodemographic groups of participants being unable to attend focus groups, purposive sampling will be conducted to target missed groups, and individual telephone interviews will be conducted, following the same semi-structured template as described above for focus groups. All interviews and focus groups will be audio-recorded and field notes taken. It is expected that at least 4 focus groups will be required per RCT site (8 in total).

\section{Objective 2: Impact evaluation}

a) Participation in falls prevention strategies

An increase in participation in falls prevention strategies is the key intended impact of the RESPOND programme. Measurement of participation will be consistent for all impact and outcome evaluation objectives and is defined as the rate of GP; physiotherapy; occupational therapy; falls clinic/ specialist; geriatrician; optometrist; and ophthalmologist appointments attended by RESPOND participants (control and intervention). This information will be captured alongside RESPOND RCT data, in the participant-completed calendars. These specific strategies were chosen as they correlate with management strategy options available to address the RESPOND modules. Estimation of participation in falls prevention strategies is powered to detect a significant difference in participation rates for falls prevention between the intervention and control groups in the 12 month follow-up. Assuming a control group participation rate of 5.7 appointments attended per personyear $[3,26]$, the minimum percentage change in participation that can be detected with $80 \%$ power at the $5 \%$ level of significance is $12.5 \%$ when taking into account the sample size for the RESPOND RCT $(n=528)$. For this we require 188 participants per group. To allow for $20 \%$ loss to follow up, 468 participants are required ( $n=234$ per group). 
Data related to specific falls prevention activities, such as Vitamin D tests, duel-energy X-ray absorptiometry (DXA) scans, and home safety assessments are not captured in the calendars as these may prompt behaviour change. In addition, as RESPOND is focused on four high level evidence-based risk factors and corresponding management strategies, participants may be involved in other falls prevention strategies beyond the scope of RESPOND. In order to capture this additional participatory data, a questionnaire will be sent to all participants (intervention and control) at 12 months. The questionnaire will ask a series of open and closed questions related to participation in a broad spectrum of falls prevention activities, including specific investigations. Perceived barriers and facilitators to participation in falls prevention activities will also be explored. Data from the 12month questionnaire will provide valuable insight into standard care available in the community. The timing of this questionnaire aims to reduce the chance of influencing participant behaviour during the trial period.

b) Participant characteristics, RESPOND programme factors, and their influence on participation in falls prevention activities

This component of the impact evaluation will identify relationships between certain participant characteristics, RESPOND programme factors, and higher or lower levels of participation in falls prevention strategies. Key participant characteristics will be identified at baseline, and will include: age; gender; lives alone; level of independence; falls risk status; falls history; employment status; comorbidities; and health literacy. Falls risk status, falls history (past 12 months) and comorbidities will be determined by the FROP-Com. Health literacy will be determined by the Health Literacy Questionnaire [35]. Whether the participant lives alone, employment status and level of independence will be determined at baseline assessment through clinician interview with the participant. Age and gender will be determined from hospital records at the point of recruitment. Exploratory analysis will be undertaken to determine if there are any other significant factors. RESPOND programme factors include the intensity, frequency and duration of intervention delivered, 
modules chosen, and goals achieved. This information will be available from the process evaluation detailed above (objective 1).Participation will be assessed by combining data pertaining to health service utilisation (as described in objective 2a).

\section{Objective 3: Outcome evaluation}

a) Participant characteristics, participatory and RESPOND programme factors and their influence on falls, fall-injuries and ED re-presentations.

Ultimately, it is arguably most important to understand who a falls prevention programme is best and least effective for, in terms of achieving trial outcomes. This allows for increased effectiveness and efficiency in future application of the programme. This sub-group analysis will identify the relationships between participant characteristics and participatory factors (as determined in the impact evaluation), RESPOND programme factors (as determined in the process evaluation) and the main outcomes for the RESPOND RCT: falls, fall injuries and ED re-presentations, in order to enhance the understanding and value of the trial results. Data related to these three trial outcomes will be obtained from RESPOND RCT data collected via participant calendars, verified with monthly phone calls from an outcome assessor, and hospital administrative data (as described above for RESPOND RCT outcomes and data collection).

\section{Data analysis and synthesis}

\section{Quantitative analysis}

The data will be analysed in two separate stages: primary and a secondary analysis. The primary analysis will compare the participation in falls prevention strategies between the control and intervention groups at six and 12 months post-baseline. Differences between groups will be compared using negative binomial regression. Secondary analyses include descriptive statistics of process measures (reach, delivery and exposure), such as mean, standard deviation, frequency and proportion to be calculated as appropriate. We will assess differences in participation, falls, fall 
injuries, and ED re-presentations across covariates by adding a treatment group by covariate interaction term to the negative binomial regression models. Covariates to be considered in the analysis are: age; gender; lives alone; level of independence; falls risk status; falls history; employment status; comorbidities; and health literacy. A variable for adjustment by site will be included in all analyses. A significance level of $p<0.05$ will be used. Stata software will be utilised to analyse quantitative data.

Qualitative analysis

Qualitative data will be transcribed and coded by two members of the research team and thematic analysis will be used to analyse the data. An inductive approach will be used to analyse focus groups, interviews, qualitative aspects of questionnaires, and free text options in recruiter and clinician notes. Both a deductive and inductive approach will be used to analyse the intervention audiorecordings. A deductive approach will be applied to determine the degree to which RESPOND clinicians adhere to key RESPOND activities, as per the logic model (Figure 1). This includes provision of education, application of motivational interviewing techniques and facilitation and coordination of services. An inductive approach will allow for identification and analysis of clinician-participant interactions beyond the scope of RESPOND. If at any stage consensus cannot be reached, a third researcher will review those aspects [36]. NVivo software will be used to facilitate management of the qualitative data and analysis.

Integration of quantitative and qualitative data

An inter-site comparison will be conducted for each objective in order to determine site-specific similarities and differences. As the trial spans two Australian states, state-specific variations, such as geographical, political and economic factors, as well as hospital specific variations such as organisational structure, funding, and culture may influence the success of the RESPOND RCT. An inter-site comparison will allow for conclusions related to the generalizability of the programme to a 
wider population. Quantitative and qualitative data will be triangulated, summarised and interpreted. The extent to which, and in what ways, results from the two types of data converge, diverge, relate to each other, and/ or produce a more complete understanding will be reported and discussed.

\section{Ethics}

Ethics approval was obtained from each of the participating hospitals, Alfred Health (HREC 439/13) and Royal Perth Hospital (REG 13-128), Monash University Human Research Ethics Committee (MUHREC CF13/3869-201300) and Curtin University HREC (HR 43/ 2014). Ethics approval covers both the RESPOND RCT and programme evaluation.

\section{DISCUSSION}

This paper details a mixed methods programme evaluation to be conducted alongside an RCT of a patient-centred falls prevention programme - RESPOND. The evaluation aims to address the lack of comprehensive multi-level evaluations conducted within the falls prevention domain. The results of this evaluation will assist in explaining the RESPOND RCT results, including sub-group analyses identifying factors associated with better or worse outcomes and who benefits most and least, in order to effectively and efficiently target limited resources for future falls prevention research and practice. Insights into the coaching style of programme delivery, including education, patientcentred decision making and motivational interviewing, will be transferable beyond the realms of falls prevention and are likely to contribute to policy and practice for a range of chronic conditions that may benefit from similar styles of coaching.

There are a number of methodological strengths to this programme evaluation. Utilising a mixed methods design which incorporates both quantitative and qualitative data allows for a richer understanding of the RESPOND programme than either method alone. Conducting the evaluation alongside an RCT has the advantage of planning for timely and appropriate data collection, in 
synergy with RCT data collection. In addition, the study design allows for comparison between the control and intervention group, adding greater value to the results than evaluation of the intervention group alone.

Triangulation of data from both the clinician and participant perspectives will allow for insights into any similarities or discrepancies between the viewpoints of those delivering and receiving the programme, increasing internal validity of the study. The use of audio-recordings of intervention contacts in addition to participant and clinician reported data will reduce the impact of recall and reporting bias, further adding to the validity of the findings. Furthermore, an inter-site comparison will facilitate conclusions related to the generalizability of the programme.

\section{CONCLUSION}

This multi-level programme evaluation will add value to the RESPOND RCT results and address the current gaps in literature related to comprehensive evaluations of RCTs of falls prevention programmes. The results of this study will inform emergency and other health service decision makers regarding implementation of policies and practice for falls prevention initiatives as well as provide valuable insights for preventive health programmes for a range of chronic conditions.

\section{Acknowledgements}

This programme evaluation would not be possible without the contributions of the RESPOND investigator team responsible for the development and implementation of the RESPOND RCT. RESPOND team members who are not co-authors in this paper, and their affiliations, are listed below:

Dr Glenn Arendts, University of Western Australia, Perth, Western Australia, Australia; Harry Perkins Institute for Medical Research, Perth, Western Australia, Australia 
Prof Peter Cameron, Health Services Research Unit, Centre of Research Excellence in Patient Safety, Division of Health Services and Global Health Research, Department of Epidemiology and Preventive Medicine, Monash University, Melbourne, Victoria, Australia

Prof Andrew Forbes, Department of Epidemiology and Preventive Medicine, Monash University, Melbourne, Victoria, Australia

A/ Prof Terrance Haines, Department of Physiotherapy, Monash University, and Allied Health Research Unit, Monash Health, Melbourne, Victoria, Australia

Dr De Villiers Smit, Alfred Health, Melbourne, Victoria, Australia

Dr Nicholas Waldron, Health Networks Branch, System Policy and Planning, Department of Health, Government of Western Australia, Perth, Western Australia, Australia

\section{Contributors}

RLM led the drafting of all sections of the article in consultation with all the coauthors. ALB led the application for funding for this work. All authors provided substantial contribution to concept and design of the programme evaluation, drafting the protocol paper and revising it critically for important intellectual content and final approval of the version to be published.

\section{Funding}

This project is funded under the Australian National Health and Medical Research Council (NHMRC) Partnership Projects funding scheme (project number APP1056802) with financial and in-kind contributions from the partner organisations listed above. $A B$ is funded by a Career Development Fellowship funded by the NHMRC (1067236), JR is funded by a Postdoctoral Fellowship co-funded by 
the NHMRC and National Heart Foundation (632933) and JAL is funded by an Early Career Fellowship funded by the NHMRC (1052442).

\section{Competing interests}

None 


\section{REFERENCES}

1. Samaras, N., et al., Older patients in the emergency department: a review. Annals of emergency medicine, 2010. 56(3): p. 261-269.

2. Bloch, F., et al., Do ED staffs have a role to play in the prevention of repeat falls in elderly patients? The American journal of emergency medicine, 2009. 27(3): p. 303-307.

3. Russell, M.A., et al., A randomized controlled trial of a multifactorial falls prevention intervention for older fallers presenting to emergency departments. Journal of the American Geriatrics Society, 2010. 58(12): p. 2265-2274.

4. Salter, A., et al., Community-dwelling seniors who present to the emergency department with a fall do not receive Guideline care and their fall risk profile worsens significantly: a 6-month prospective study. Osteoporosis international, 2006. 17(5): p. 672-683.

5. Scheffer, A.C., et al., Fear of falling: measurement strategy, prevalence, risk factors and consequences among older persons. Age and ageing, 2008. 37(1): p. 19-24.

6. Gillespie, L.D., et al., Interventions for preventing falls in older people living in the community. Cochrane Database Syst Rev, 2012. 9(11).

7. AlHW: Bradley, C., Trends in hospitalisations due to falls by older people, Australia: 1999-00 to 2010-11. Injury research and statistics no. 84. Cat. no. INJCAT 160, A.I.o.H.a. Welfare, Editor. 2013, AlHW: Canberra.

8. Kannus, P., et al., Fall-induced deaths among elderly people. American journal of public health, 2005. 95(3): p. 422.

9. Deandrea, S., et al., Risk factors for falls in community-dwelling older people: a systematic review and meta-analysis. Epidemiology, 2010. 21(5): p. 658-68.

10. Bloch, F., et al., Episodes of falling among elderly people: a systematic review and metaanalysis of social and demographic pre-disposing characteristics. Clinics, 2010. 65(9): p. 895903.

11. Shubert, T.E., et al., Complexities of Fall Prevention in Clinical Settings: A Commentary. The Gerontologist, 2013.

12. Australian Commission on Safety and Quality in Health Care, Preventing Falls and Harm From Falls in Older People: Best Practice Guidelines for Australian Community Care. 2009, Commonwealth of Australia: Australia.

13. National Institute for Health and Care Excellence, Falls: assessment and prevention of falls in older people. 2013, NICE: UK.

14. Nyman, S.R. and C.R. Victor, Older people's participation in and engagement with falls prevention interventions in community settings: an augment to the Cochrane systematic review. Age and ageing, 2012. 41(1): p. 16-23.

15. Haines, T.P., et al., "Better for others than for me": A belief that should shape our effeorts to promote participation in falls prevention strategies. Archives of Gerontology and Geriatrics, 2014.

16. Bunn, F., et al., A systematic review of older people's perceptions of facilitators and barriers to participation in falls-prevention interventions. Ageing and Society, 2008. 28(04): p. 449472.

17. Manning, D. and C. Dickens, Health literacy: more choice, but do cancer patients have the skills to decide? European journal of cancer care, 2006. 15(5): p. 448-452.

18. Bush, R.A., F.M. Boyle, and R. Ostini, Risks associated with low functional health literacy in an Australian population. Med. J. Aust., 2010. 192(8): p. 479-479.

19. Oakley, A., et al., Process evaluation in randomised controlled trials of complex interventions. Bmj, 2006. 332(7538): p. 413-416.

20. Craig, P., et al., Developing and evaluating complex interventions: the new Medical Research Council guidance. Bmj, 2008. 337. 
21. Carroll, C., et al., A conceptual framework for implementation fidelity. Implementation Science, 2007. 2(40): p. 1-9.

22. Hasson, H., Systematic evaluation of implementation fidelity of complex interventions in health and social care. Implement Sci, 2010. 5(1): p. 67.

23. Deery, H.A., L.M. Day, and B.N. Fildes, An impact evaluation of a falls prevention program among older people. Accident Analysis \& Prevention, 2000. 32(3): p. 427-33.

24. Nutbeam, D., Evaluating health promotion-progress, problems and solutions. Health promotion international, 1998. 13(1): p. 27-44.

25. Bleijlevens, M.H., et al., Process factors explaining the ineffectiveness of a multidisciplinary fall prevention programme: a process evaluation. BMC Public Health, 2008. 8: p. 332.

26. de Vries, O.J., et al., Multifactorial intervention to reduce falls in older people at high risk of recurrent falls: a randomized controlled trial. Archives of internal medicine, 2010. 170(13): p. 1110-1117.

27. Nikolaus, T. and M. Bach, Preventing falls in community - dwelling frail older people using a home intervention team (HIT): results from the randomized Falls - HIT trial. Journal of the American Geriatrics Society, 2003. 51(3): p. 300-305.

28. Creswell, J.W. and V.L.P. Clark, Designing and conducting mixed methods research. 2007: Wiley Online Library.

29. Barker, A., et al., RESPOND-A patient-centred program to prevent secondary falls in older people presenting to the emergency department with a fall: Protocol for a multi-centre randomised controlled trial. Injury Prevention, 2014.

30. WHO. WHO Global Report on Falls Prevention in Older Age. . Secondary WHO Global Report on Falls Prevention in Older Age 2007; Available from:

http://www.who.int/violence injury prevention/other injury/falls/en/index.html

31. Steckler, A.B., L. Linnan, and B. Israel, Process evaluation for public health interventions and research. 2002: Jossey-Bass San Francisco.

32. Shields, C.G., et al., Rochester participatory decision-making scale (RPAD): reliability and validity. The Annals of Family Medicine, 2005. 3(5): p. 436-442.

33. Russell, M.A., et al., The reliability and predictive accuracy of the falls risk for older people in the community assessment (FROP-Com) tool. Age and ageing, 2008. 37(6): p. 634-639.

34. Russell, M.A., et al., Development of the Falls Risk for Older People in the Community (FROPCom) screening tool*. Age and ageing, 2009. 38(1): p. 40-46.

35. Osborne, R.H., et al., The grounded psychometric development and initial validation of the Health Literacy Questionnaire (HLQ). BMC public health, 2013. 13(1): p. 658.

36. Vaismoradi, M., H. Turunen, and T. Bondas, Content analysis and thematic analysis: Implications for conducting a qualitative descriptive study. Nursing \& health sciences, 2013. 15(3): p. 398-405. 
Figure 1: RESPOND programme logic model

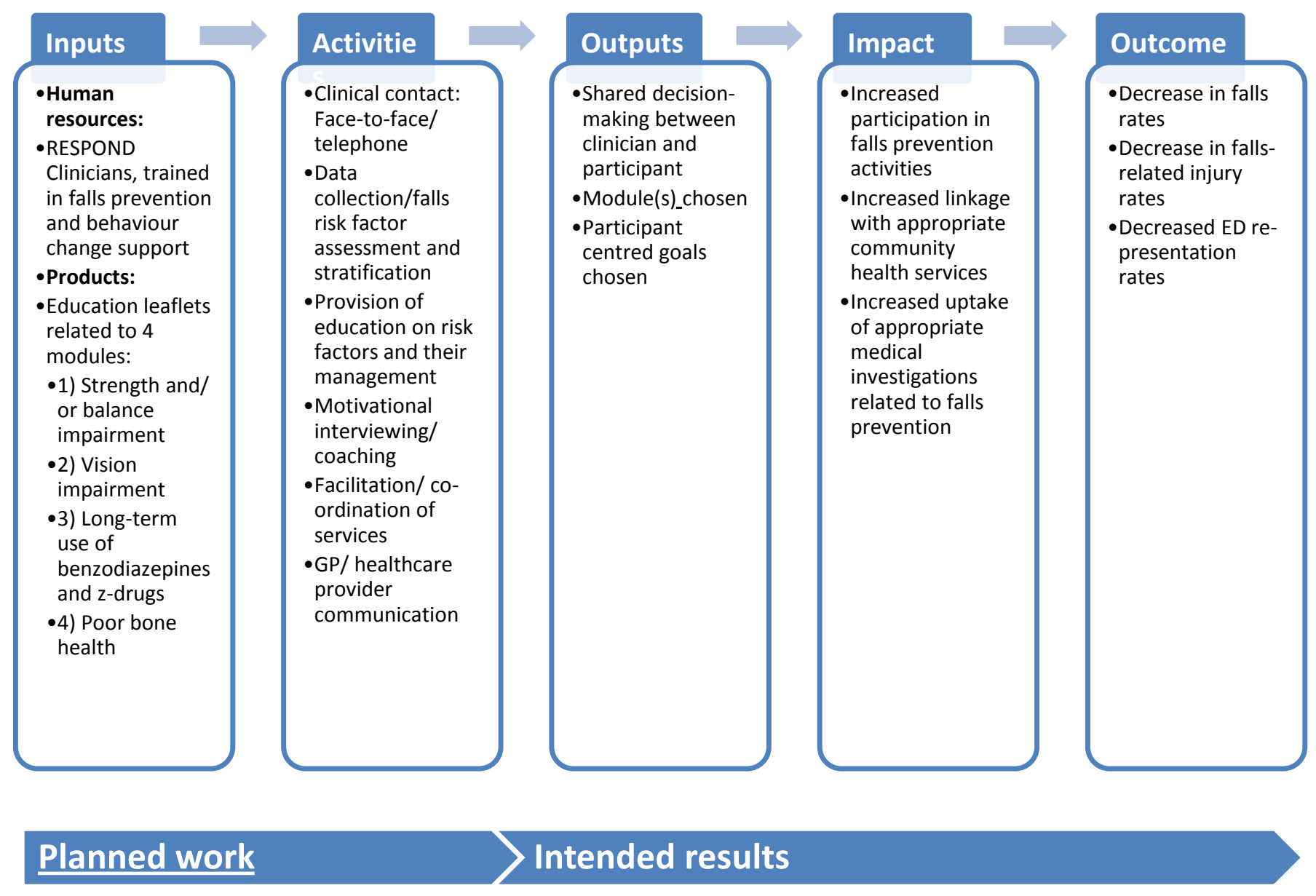

Notes: $\mathrm{ED}=$ Emergency department; $\mathrm{GP}=$ General practitioner 
Table 1: RESPOND program evaluation plan

\begin{tabular}{|c|c|c|c|}
\hline Objective & Evaluation component & Data source & $\begin{array}{l}\text { Timing of data } \\
\text { collection }\end{array}$ \\
\hline \multicolumn{4}{|l|}{ 1. Process evaluation } \\
\hline \multirow{6}{*}{$\begin{array}{l}\text { a) Assess the degree to which } \\
\text { RESPOND was implemented as } \\
\text { planned. }\end{array}$} & \multirow[t]{2}{*}{ Implementation fidelity: Reach } & Recruiter records & Recruitment \\
\hline & & Hospital administrative data & 12 months \\
\hline & \multirow[t]{2}{*}{ Implementation fidelity: Content } & Clinician records & 6 months \\
\hline & & Audio-recordings of clinician -participant contacts & 6 months \\
\hline & Implementation fidelity: Dosage & Clinician records & 6 months \\
\hline & Implementation fidelity: Exposure & Clinician records & 6 months \\
\hline \multirow{5}{*}{$\begin{array}{l}\text { b) Identify barriers and } \\
\text { facilitators to implementation } \\
\text { from the perspectives of those } \\
\text { delivering and receiving the } \\
\text { intervention. }\end{array}$} & \multirow{3}{*}{$\begin{array}{l}\text { Barriers and facilitators, acceptability, } \\
\text { perceived relevance, perceived benefit }\end{array}$} & Participant questionnaire (intervention) & 6 months \\
\hline & & Participant focus groups (intervention) & 6 months \\
\hline & & RESPOND clinician interviews & 12 months \\
\hline & $\begin{array}{l}\text { Barriers and facilitators to achieving } \\
\text { RESPOND goals }\end{array}$ & Clinician records & 6 months \\
\hline & RESPOND clinician training and support & RESPOND clinician interviews & 12 months \\
\hline \multicolumn{4}{|l|}{ 2. Impact evaluation } \\
\hline \multirow{2}{*}{$\begin{array}{l}\text { a) Identify whether RESPOND } \\
\text { increases participation in falls } \\
\text { prevention strategies, and } \\
\text { factors influencing participation, } \\
\text { among the intervention group, } \\
\text { compared with standard care. }\end{array}$} & \multirow[t]{2}{*}{$\begin{array}{l}\text { Participation in falls prevention } \\
\text { strategies }\end{array}$} & $\begin{array}{l}\text { Participant-completed calendars (intervention and } \\
\text { standard care) }\end{array}$ & 6 and 12 months \\
\hline & & $\begin{array}{l}\text { Participant questionnaire (intervention and } \\
\text { standard care) }\end{array}$ & 12 month \\
\hline \multirow{6}{*}{$\begin{array}{l}\text { b) Determine the degree to } \\
\text { which participant characteristics } \\
\text { and RESPOND programme } \\
\text { factors are associated with } \\
\text { participation in falls prevention } \\
\text { strategies, among the } \\
\text { intervention group. }\end{array}$} & \multirow[t]{4}{*}{ Participant characteristics } & Hospital administrative data & Baseline \\
\hline & & FROP-Com & Baseline \\
\hline & & Health Literacy Questionnaire & Baseline \\
\hline & & Initial clinician interview with participant & Baseline \\
\hline & Programme factors & As per objective $1 \mathrm{a}$ & 6 months \\
\hline & $\begin{array}{l}\text { Participation in falls prevention } \\
\text { strategies }\end{array}$ & As per objective $2 a$ & 6 and 12 months \\
\hline \multicolumn{4}{|l|}{ 3. Outcome evaluation } \\
\hline \multirow{6}{*}{$\begin{array}{l}\text { a) Determine the degree to } \\
\text { which participant characteristics, } \\
\text { participatory and RESPOND } \\
\text { programme factors influence } \\
\text { falls, fall-injuries and ED re- } \\
\text { presentations. }\end{array}$} & Participant characteristics & As per objective $2 b$ & Baseline \\
\hline & $\begin{array}{l}\text { Participation in falls prevention } \\
\text { strategies }\end{array}$ & As per objective $2 a$ & 6 and 12 months \\
\hline & Programme factors & As per objective $1 \mathrm{a}$ & 6 months \\
\hline & Falls and falls injuries & $\begin{array}{l}\text { Participant-completed calendars (intervention and } \\
\text { standard care) }\end{array}$ & 6 and 12 months \\
\hline & \multirow[t]{2}{*}{ ED re-presentations } & $\begin{array}{l}\text { Participant-completed calendars } \\
\text { (intervention and standard care) }\end{array}$ & 6 and 12 months \\
\hline & & Hospital administrative data & 12 months \\
\hline
\end{tabular}

